\title{
Adaptation and National Dissemination of a Brief, Evidence-Based, HIV Prevention Intervention for High-Risk Men Who Have Sex with Men
}

\author{
Jeffrey H. Herbst, PhD \\ Jerris L. Raiford, PhD \\ Monique G. Carry, PhD \\ Aisha L. Wilkes, MPH \\ Renata D. Ellington, MS Ed \\ David K. Whittier, PhD \\ National Center for HIVIAIDS, Viral Hepatitis, STD, and TB Prevention, CDC
}

Corresponding author: Jeffrey H. Herbst, Division of HIV/AIDS Prevention, National Center for HIV/AIDS, Viral Hepatitis, STD, and TB Prevention, CDC. Telephone: 770-488-3779; E-mail: jherbst@cdc.gov.

\section{Summary}

CDC's high-impact human immunodeficiency virus (HIV) prevention approach calls for targeting the most cost-effective and scalable interventions to populations of greatest need to reduce HIV incidence. CDC has funded research to adapt and demonstrate the efficacy of Personalized Cognitive Counseling (PCC) as an HIV prevention intervention. Project ECHO, based in San Francisco, California, during 2010-2012, involved an adaptation of PCC for HIV-negative episodic substance-using men who have sex with men (SUMSM) and a randomized trial to test its efficacy in reducing sexual and substance-use risk behaviors. Episodic substance use is the use of substances recreationally and less than weekly. PCC is a 30-minute to 50-minute counseling session that involves addressing self-justifications men use for engaging in risky sexual behavior despite knowing the potential for HIV infection. By exploring these justifications, participants become aware of the ways they make sexual decisions, become better prepared to realistically assess their risk for HIV during future risky situations, and make decisions to decrease their HIV risk. The findings of Project ECHO demonstrated the efficacy of PCC for reducing HIV-related substance-use risk behaviors. The study also demonstrated efficacy of PCC for reducing sexual risk behaviors among SUMSM screened as nondependent on targeted drug substances. CDC has identified PCC as a "best evidence" HIV behavioral intervention and supports its national dissemination. Several features of PCC enhance its feasibility of implementation: it is brief, delivered with HIV testing, relatively inexpensive, allows flexibility in counselor qualifications and delivery settings, and is individualized to each client. The original PCC and its adapted versions can contribute to reducing HIV-related health disparities among high-risk MSM, including substance users, by raising awareness of and promoting reductions in personal risk behaviors.

\section{Introduction}

Gay, bisexual, and other men who have sex with men (MSM) are affected disproportionately by human immunodeficiency virus (HIV) in the United States. Although approximately 3\% of the adolescent and adult U.S. male population is estimated to have engaged in same-sex behavior in the past year (1), in 2011, MSM accounted for $65 \%$ of the estimated 49,273 new HIV infections and $82 \%$ of the estimated 38,825 HIV diagnoses among all males aged $\geq 13$ years (2). Sexual risk behavior accounts for most HIV infections among MSM, and anal intercourse without a condom is the primary route for transmitting HIV infection to an uninfected person.

Over the past decade, the United States has demonstrated improvements in preventing new HIV infections. During 2002-2011, the annual HIV diagnosis rate decreased by $33.2 \%$ overall (from 24.1 per 100,000 population in 2002 to 16.1 per 100,000 population in 2011 ), with statistically significant decreases reported among women, persons aged
35-44 years, persons of multiple races, and injecting drug users (3). Although the annual number of HIV diagnoses during 2002-2011 remained stable for MSM overall, those aged 13-24 years and $\geq 45$ years experienced increases in HIV diagnoses (3). MSM aged 13-24 years experienced a 133\% increase in HIV diagnoses during that time period.

Racial/ethnic minority MSM are particularly affected by HIV. In 2011, black MSM accounted for an estimated 38\% of all new HIV infections among all U.S. men, compared with an estimated 34\% among white MSM (2). During 2008-2010, HIV infections increased 20\% among young black MSM aged 13-24 years, and HIV infections increased $39 \%$ among Hispanic/Latino MSM aged 25-34 years (4). In contrast, increases in HIV infections during the same period were lower among white MSM aged 13-24 years and aged $25-34$ years (18\% and 20\%, respectively) (4). In addition, in 2011, HIV continued to be listed among the top 10 leading causes of death among all U.S. men aged 25-54 years (5). Together these data indicate that improving the effectiveness 
of HIV prevention efforts for MSM in the United States is a critical public health goal (G).

Many MSM experience HIV-related disparities in access to and receipt of medical care $(7,8)$. Analysis of data from the 2006-2010 National Survey of Family Growth indicated that sexually active MSM (i.e., men who reported having sex with another man in the past 12 months) reported suboptimal rates of preventive services for HIV and other sexually transmitted diseases (STDs), including screening for infection and receiving behavioral counseling to reduce risk behaviors, as compared with men who only have sex with women (9). According to a National HIV Behavioral Surveillance survey of approximately 8,000 MSM, $11 \%$ of respondents selfreported being HIV-positive; among these HIV-positive men, $25 \%$ reported delayed linkage to HIV medical care, and 12\% reported receiving no HIV care, and of those reporting at least one health care visit, $30 \%$ were not receiving life-sustaining antiretroviral medication (10). Racial/ethnic minority MSM report significantly lower rates of HIV testing and, if they test HIV-positive, lower rates of linkage to HIV medical care, retention in HIV medical care, access to antiretrovirals, and HIV viral suppression than their white counterparts (11-14). Data reported in 2010 from CDC's National HIV Surveillance System and Medical Monitoring Project indicated that black MSM, compared with white and Hispanic/Latino MSM, experience lower levels of linkage to HIV medical care (71.6\% blacks versus $82.9 \%$ whites and $80.3 \%$ Hispanics/Latinos), retention in HIV medical care $(46.3 \%$ versus $52.1 \%$ and $54.1 \%$ ), access to antiretroviral prescriptions ( $47.1 \%$ versus $49.6 \%$ and $49.2 \%$ ), and HIV viral suppression (37.0\% versus $43.9 \%$ and $41.5 \%$ ) (15). These data indicate increasing access to HIV care and treatment is critical for improving health outcomes and reducing HIV transmission among MSM.

HIV-related health disparities experienced by some MSM might be due to individual, interpersonal, community, and societal factors that can hamper access to health care and treatment. For some MSM, personal experiences of stigma, discrimination, and homophobia regarding their sexual orientation can result in adverse mental health outcomes, including depression, anxiety, elevated stress, and suicidal thoughts and attempts (16-21). Discrimination by health care providers (22), limited access to gay-friendly health services (23), and barriers to health insurance (23-25) also can contribute to HIV-related disparities experienced by MSM. The physical and mental health of MSM can be affected by syndemic conditions, which are conditions that individually or in combination can increase HIV risk. These syndemic conditions include, but are not limited to, substance use and abuse, mental health problems, prevalence of STDs in the community, poverty, unemployment, and experiences of emotional and physical violence and abuse ( 7 ).

National survey data suggest that many MSM consume alcohol and other drugs that can impair judgment and increase risky behavior $(26,27)$. Among MSM populations, methamphetamine, amyl nitrate (poppers), cocaine, and heavy alcohol use (i.e., binge drinking) are the substances most consistently associated with risky sexual behavior (28-30) and increased HIV risk (31). Many substance-using MSM (SUMSM) use these substances episodically* or recreationally, and many of these men might not be aware of their level of risk (32).

On the basis of the disproportionate occurrence of HIV among MSM and the national priority to reduce new infections among these men (33), evidence-based behavioral interventions are needed to reduce HIV-related sexual and substance-use risk behaviors. Few HIV prevention interventions exist for MSM (34), and of those that do exist, none have proven efficacy for reducing risky sex with concomitant substance use $(30,35)$. Personalized Cognitive Counseling (PCC) is an evidence-based behavioral intervention that reduced events of anal sex without condoms among HIV-negative MSM in two efficacy trials. In one trial the intervention was delivered by professional mental health counselors (36), and in the second trial the intervention was delivered by paraprofessional counselors (37). Secondary analysis of data in a 2007 trial indicated PCC reduced events of anal sex without condoms among men of color, including men who were black, Hispanic/Latino, Asian, and of mixed race/ ethnicity (38). A third trial testing the efficacy of a two-session PCC adapted for HIV-infected MSM did not show efficacy (39). Together these studies indicate PCC is an efficacious behavioral intervention for reducing sexual risk behavior (anal sex without condoms) among HIV-negative MSM.

PCC involves a brief counseling session for addressing selfjustifications men use for engaging in risky behavior despite knowing the potential for HIV infection. By exploring selfjustifications used for increased risky behavior, participants become aware of the ways they make decisions about sex, become better prepared to realistically assess their risk for acquiring HIV during future risky situations, and make decisions that decrease their HIV risk (40). PCC is delivered in conjunction with HIV testing and can contribute to reducing HIV-related health disparities among MSM by raising awareness of and promoting reductions in personal risk behaviors. The counseling session, HIV testing service, and referral process emphasize the importance of HIV prevention among men and their sex partners.

\footnotetext{
* Episodic substance use is the use of substances recreationally and less than weekly.
} 
This report summarizes published findings of a CDC-funded research study to adapt and demonstrate the efficacy of the adapted PCC for reducing HIV-related risk behaviors among a high-risk population of episodic SUMSM. The report also describes efforts by CDC to translate the PCC curriculum into a package of user-friendly materials and to disseminate the intervention throughout the nation.

CDC's Office of Minority Health and Health Equity selected the intervention analysis and discussion that follows to provide an example of a program that might be effective in reducing HIV-related health disparities affecting high-risk MSM, including substance users. Criteria for selecting this program are described in the Background and Rationale for this supplement (41).

\section{Methods}

\section{Intervention Methods}

In 2007, CDC funded Public Health Foundation Enterprises to conduct a research study in collaboration with the San Francisco Department of Public Health (42). The study, Project ECHO, involved a systematic adaptation of PCC for HIV-negative episodic SUMSM (43) and a randomized trial to test the efficacy of the adapted PCC in reducing sexual and substance-use risk behaviors in this population at high risk for HIV $(44,45)$. Episodic substance use was defined as use of substances recreationally and less than weekly.

PCC is a 30-minute to 50-minute counseling session delivered in conjunction with routine HIV testing. MSM clients work with a trained counselor to appraise their personal HIV risk and discuss less risky alternatives for future behavior. PCC is delivered in five steps: 1) the client recollects a memorable event of anal intercourse without a condom (or unprotected anal intercourse [UAI]), 2) the client completes a questionnaire containing self-justifications he might have used during the UAI event, 3) the counselor helps the client draw out the story of the memorable UAI event, 4) the counselor helps the client identify self-justifications that facilitated the UAI, and 5) the client decides how to handle future risky situations (Figure).

PCC was adapted for episodic SUMSM by focusing on selfjustifications for engaging in UAI and concurrent substance use. The adapted PCC questionnaire included 17 items retained from the original PCC and 31 newly developed items specific to episodic SUMSM (43). A list of the most common self-justifications endorsed by SUMSM and details regarding the adaptation are reported elsewhere (43).
FIGURE. Five steps to implementing PCC risk-reduction intervention for men who have sex with men

Step 1. Counselor asks client to recall a recent memorable event of UAI.

Purpose: After the client is determined to be eligible for PCC, the counselor asks him to think of a recent memorable event of UAI. Through conversation, the counselor helps the client identify an appropriate incident.

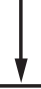

Step 2. Counselor administers PCC questionnaire.

Purpose: Once an appropriate incident is identified, the counselor asks the client to complete the PCC questionnaire with the specific event in mind.

\section{thoughts and feelings.}

Purpose: The counselor helps the client tell the whole story of the recent event of UAI-what led up to it, what he did, what happened afterward, and how he felt about it. As the client tells his story, the counselor asks what his thoughts and feelings were before, during, and after.

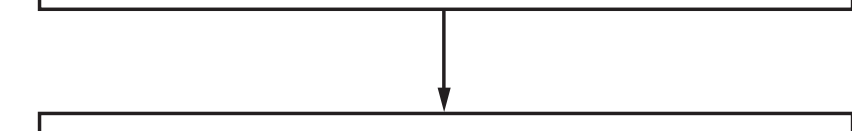

Step 4. Counselor identifies and discusses the self-justification(s) with client.

Purpose: While listening for any self-justifications for UAl, such as "It just happened, I didn't mean to," the counselor asks the client how and to what extent he thought about HIV transmission during the event. The counselor asks the client what he thinks now about the self-justifications that were in his mind during the UAl event.

Purpose: After the story has been told and the client has reflected on his thoughts and feelings, the counselor asks the client what he thinks will happen in the future, what he thinks he will do in a similar situation, and how he might approach it differently. The counselor supports the client's constructive plans.

Source: Adapted from University of California San Francisco, AIDS Health Project. Personalized cognitive counseling implementation manual. San Francisco, CA: University of California San Francisco; 2011. https://effectiveinterventions.cdc. gov/docs/default-source/pcc-docs/13-0204_PCC_Final_IM_Appendices_and_ Workbook.pdf?sfvrsn $=0$.

Abbreviations: $\mathrm{PCC}=$ Personalized Cognitive Counseling; UAI = unprotected anal intercourse. 


\section{Data Collection and Analysis}

The randomized trial occurred during May 2010-May 2012 in San Francisco. Episodic SUMSM who reported engaging in UAI while under the influence of alcohol and other drugs were recruited via street outreach at community venues frequented by MSM (e.g., outside of bars, clubs, gyms, and grocery stores). To recruit a racially and ethnically diverse sample, recruitment also occurred at community-based organizations serving black, Latino, and Asian/Pacific Islander MSM.

Eligible MSM included those reporting UAI with men while under the influence of one or any combination of substances (methamphetamine, poppers, crack or powder cocaine, or alcohol [if binge drinking]) 2 hours before or during sex within the past 6 months. Men were assigned randomly to either the intervention group (i.e., adapted PCC plus a rapid HIV test; $n=162$ ) or the control group (i.e., rapid HIV test only; $\mathrm{n}=164)$. All participants completed assessments at baseline and at 3-and 6-month follow-up, received a rapid HIV test following CDC's guidelines for HIV testing in health care settings (46), and received monetary incentives for participation. PCC participants received a booster intervention session after completing the 3-month follow-up assessment. Details regarding the adapted intervention and study methods are reported elsewhere (43). All study activities took place at the San Francisco Department of Public Health, and the University of California Committee on Human Research approved the study protocol.

Sexual risk outcomes reported during the past 3 months included number of UAI events (by type [insertive or receptive] and total number), number of UAI partners, number of UAI events with three most recent nonprimary partners, number of serodiscordant UAI events, and number of condom-protected anal intercourse events. Substance-use outcomes during the past 3 months included use of the following substances: alcohol when binge drinking (i.e., five or more drinks per occasion), ecstasy, gamma-hydroxybutyrate (GHB), marijuana, methamphetamine, poppers, crack, cocaine, prescription opioids (codeine, Vicodin, and OxyContin), and erectile dysfunction drugs (Viagra, Levitra, and Cialis). Substance use concurrent with sexual risk outcomes (e.g., number of UAI events) during the past 3 months were also assessed. Baseline self-reported substance dependence for methamphetamines, poppers, cocaine, and alcohol were defined according to the Severity of Dependence Scale (47).

Intervention efficacy was evaluated using generalized estimating equation models to test group-specific linear trends on outcomes across three study visits: baseline and 3- and 6-month follow-ups. Overall, 96\% of participants completed the 3-and 6-month follow-up assessments. Intervention efficacy analyses were stratified according to substance dependence. For analyses assessing substance-use outcomes, event-level data were collected on substances used within 2 hours before/ during UAI events. Intent-to-treat analyses were conducted according to random allocation to study arm and including all observed study data. Logistic (binary outcomes) and negative binomial (events outcomes) generalized estimating equation models with robust standard errors were used to evaluate linear trends in alcohol and substance-use outcomes throughout the 6 months of follow-up. Economic or cost data were not assessed in this study.

\section{Results}

Among the 326 participants, 47\% were white and 53\% were nonwhite, including 26\% Hispanic/Latino, 11\% Asian/ Pacific Islander, 10\% black, and 6\% mixed or other race (44). The mean age of participants was 33.6 years. At baseline, $89 \%$ of participants self-reported binge drinking, $42 \%$ reported popper use, $34 \%$ cocaine use (powder or crack), and $10 \%$ methamphetamine use. A total of 138 participants ( $42 \%$ of the 326 SUMSM) were classified as substance dependent based on the Severity of Dependence Scale, including 7 men for methamphetamines, 12 for poppers, 21 for cocaine, and 122 for alcohol. Among the total sample of 326 participants, two tested HIV-positive at the baseline visit, three at the 3-month visit, and none at the 6 -month visit. No statistically significant between-group differences were found in the overall sample of episodic SUMSM on demographic characteristics; the primary sexual risk outcomes of number of UAI events, number of UAI partners, and number of UAI events with three most recent nonprimary partners; and the primary substance-use outcomes including use of methamphetamines, poppers, cocaine (powder or crack), and binge drinking.

PCC participants exhibited a trend for greater reductions in number of receptive UAI events over the entire study period than control participants (rate ratio $[R R]$ : 0.57; 95\% confidence interval $[\mathrm{CI}]: 0.33-1.01)$ (44). A planned subgroup analysis of 188 nondependent, episodic SUMSM (93 PCC and 95 control) found a $44 \%$ greater reduction in the number of UAI events with three most recent partners among PCC participants relative to controls (RR: 0.56; 95\% CI: $0.34-0.92$ ) over the study period. In subgroup analyses of nondependent SUMSM of color (i.e., men who were black, Hispanic/Latino, Asian, or mixed race/ethnicity) (44 PCC and 51 control), PCC participants reported a $59 \%$ greater reduction in total number of UAI events (RR: $0.41 ; 95 \%$ CI: $0.18-0.95)$ and $63 \%$ greater reduction in number of UAI events with three most recent nonprimary partners (RR: 0.37 ; 
95\% CI: 0.16-0.87) than controls over the study period. No significant intervention effects were found among the 138 substance-dependent participants.

For substance-use outcomes in the total sample of 326 SUMSM (45), PCC participants reported significantly greater rates of abstinence from alcohol (RR: 0.93; 95\% CI: 0.89-0.97), abstinence from marijuana (RR: 0.84; $95 \%$ CI: $0.73-0.98)$, and abstinence from erectile dysfunction drugs (RR: 0.51 ; 95\% CI: 0.33-0.79) than controls over the study period (44). In addition, PCC participants reported a $46 \%$ greater reduction in frequency of alcohol intoxication (RR: 0.54; 95\% CI: 0.34-0.85) and a 74\% greater reduction in mean number of UAI events while under the influence of methamphetamine (RR: 0.26 ; 95\% CI: 0.08-0.84) than controls over the study period.

\section{Discussion}

On the basis of the evidence of two randomized trials (36-38), CDC identified PCC as a "best evidence" HIV behavioral intervention. The intervention is listed in CDC's online Compendium of Evidence-Based Interventions and Best Practices for HIV Prevention (48). The findings of Project $\mathrm{ECHO}$, a rigorous evaluation of the adapted PCC intervention for episodic SUMSM, further demonstrated the efficacy of PCC for reducing HIV-related substance-use risk behaviors (45). The study also demonstrated efficacy of PCC for reducing sexual risk behaviors among SUMSM who are not dependent on targeted substances (44).

These findings add to a growing body of evidence that PCC reduces risk behaviors among HIV-negative MSM (36-38). The delivery of PCC in conjunction with HIV testing is critical for high-risk MSM who might be unaware of their HIV serostatus. The intervention delivered within this context can also support linkage of these high-risk HIV-negative MSM to other HIV prevention programs and services, like antiretroviral preexposure prophylaxis (PrEP) for HIV prevention $(49,50)$.

In 2007, CDC funded the development of a user-friendly intervention package of the original PCC (51). The package includes a starter kit, implementation manual and workbook, cost estimate worksheet, and guidance on technical assistance (52). These materials are available online for use by HIV prevention providers (53). A PCC training curriculum and an agency manager training program are also available online (54).

Since 2011, CDC has supported the national dissemination of PCC to agencies serving high-risk, HIV-negative MSM. To maximize the impact of HIV prevention in the United States, CDC redirected approximately $\$ 20$ million in fiscal year 2014 HIV prevention funds to better align community-based organizations (CBOs) activities with CDC's high-impact HIV prevention approach (55). CBO resources were redirected toward high-impact HIV prevention strategies and interventions, including HIV testing, linkage to and engagement and retention of HIV-diagnosed persons in medical care, and use of evidence-based behavioral interventions that are scalable, cost-effective, and scientifically proven (56). PCC is one of nine behavioral interventions for high-risk, HIV-negative persons prioritized by CDC for implementation by CBOs (57).

As of August 2014, of the 48 CBOs funded by CDC under three separate funding opportunity announcements to deliver PCC, 43 (90\%) are delivering PCC to MSM and transgender persons. These CBOs are located in 19 states, the District of Columbia, Puerto Rico, and the U.S. Virgin Islands and serve MSM and transgender persons of diverse races and ethnicities. Additional HIV prevention programs are funded by U.S. state, local, and territorial health departments to implement PCC.

During February 2011-June 2014, a total of 932 persons, representing 344 HIV prevention service organizations, completed one of 67 trainings for counselors offered by CDC on the PCC curriculum. These 932 persons represent 155 CBOs, 48 health departments, and 141 other agencies, including health clinics, private practices, and universities and represent various occupations (e.g., health educators, substance abuse counselors, clergy/faith-based counselors, and social workers). Persons attending PCC trainings serve diverse MSM and transgender populations at high risk for HIV infection, including incarcerated and paroled persons, homeless persons, persons who abuse substances, sex workers, and refugees and migrants. On the basis of the results of Project ECHO, the San Francisco Department of Public Health is supporting implementation of the adapted PCC to address binge drinking among MSM (D. Geckeler, San Francisco Department of Public Health, personal communication, July 2014).

\section{Feasibility}

Several features of PCC enhance the feasibility of its implementation. The intervention is brief, delivered with HIV testing, and of relatively low cost (i.e., estimated $\$ 145$ per client served) (58). These features make the intervention attractive to diverse HIV prevention agencies. As a singlesession intervention, issues of client engagement and retention commonly associated with multisession interventions are minimized (59). Because of the brevity of PCC, counselors only attend 2 days of classroom training, which reduces the amount of time agency staff are away from the office. In addition, as demonstrated by Project ECHO, clients might receive more 
than one PCC session that can be tailored to address persistent risky behavior.

PCC also allows for flexibility in counselor qualifications and delivery setting. PCC has demonstrated efficacy when delivered by both professionals (36) and paraprofessionals $(37,44)$. The intervention can also be conducted in clinical and nonclinical settings as long as confidentiality can be assured. Because PCC is frequently conducted within HIV testing programs, existing HIV test counselors can be trained to screen for and conduct the intervention.

HIV behavioral interventions that can be combined with effective biomedical interventions (e.g., antiretroviral PrEP for HIV prevention) are critically needed (60). The feasibility of implementing PCC with HIV testing suggests the intervention can help screen MSM at substantial risk for acquiring HIV infection who are likely to benefit from PrEP.

CDC is committed to sustaining the implementation of high-impact, evidence-based, behavioral interventions, including PCC. The CDC approach involves eight key activities: 1) planning dissemination of interventions with both researchers and public health stakeholders; 2) marketing interventions to encourage customer choice; 3) supporting changes to policies regarding funding announcements, clinical guidelines, and program guidance that support evidencebased practices; 4) disseminating intervention resources; 5) providing intervention training; 6) building capacity for and providing technical assistance with the intervention; 7) improving quality; and 8) evaluating dissemination (61). CDC funds capacity-building assistance providers to support PCC implementation.

PCC is flexible in addressing the risk-reduction needs of diverse clients because each session is individualized and addresses the client's unique thought process during a recent and memorable risky event. Moreover, PCC efficacy trials demonstrated the intervention remained efficacious among MSM of various races/ethnicities $(39,44)$. Implementation funding from CDC has resulted in applications of the intervention in new populations and contexts. For example, guidance is available on adapting the original PCC curriculum for transgender women who have sex with men (62). Implementation materials are also available in Spanish. CDC supports technical assistance on adapting PCC for different populations and settings.

\section{Limitations}

The Project ECHO efficacy study is subject to at least three limitations. First, similar to many behavioral intervention trials, intervention efficacy might be over- or under-estimated because behavior might not result in significant reductions in incidence of STDs including HIV infection. Second, sexual risk and substance-use behaviors were self-reported and might be subject to recall and social-response biases. Third, the PCC efficacy trials, including Project ECHO, were conducted in San Francisco, California, and might not be generalizable to other U.S. jurisdictions. San Francisco is a city with a long history of HIV infection among MSM, strong community norms regarding risk behavior, and well-established partnerships between the health department, community members, and local organizations, which allows for the design and delivery of effective HIV prevention programs and services (63). However, HIV surveillance data reported by the San Francisco Department of Public Health for 2013 indicated that newly diagnosed white MSM cases declined from 2006 to 2013, but new diagnoses among Hispanic/Latino, African American, and other racial/ethnic groups of MSM were fairly stable during this same period (64). Additional studies are needed to demonstrate the effectiveness and generalizability of PCC among diverse MSM populations, delivery settings, and geographic regions.

The PCC intervention is subject to at least one limitation, the brevity of the 30-minute to 50-minute counseling session. Although brief behavioral counseling might be appropriate for most at-risk MSM, some MSM might require intensive riskreduction counseling and treatment. Importantly, the findings of Project ECHO suggest PCC might not be appropriate for MSM who are dependent on alcohol and other drugs. Moreover, one efficacy trial suggests PCC is not appropriate for reducing risky sexual behavior among MSM living with HIV infection (39).

\section{Conclusion}

Although MSM continue to be affected disproportionately by HIV infection in the United States, evidence-based behavioral interventions for these men remain limited (34). The efficacy of the adapted PCC intervention for reducing HIV-related risk behaviors in a group of white and nonwhite episodic SUMSM in San Francisco, particularly among those who were nondependent episodic SUMSM, has potential for public health impact within the context of persistent rates of HIV infection among MSM. PCC is an efficacious brief counseling intervention designed to reduce HIV-related risk behaviors among diverse groups of MSM. The adapted PCC for episodic SUMSM is the only behavioral intervention with proven efficacy to reduce substance-use risk behaviors among MSM. In addition, PCC is an important component of a comprehensive HIV prevention program for high-risk MSM and is consistent with the goals of the National HIV/ 
AIDS Strategy (33) and CDC's high-impact HIV prevention approach (56). PCC can be delivered to diverse populations of MSM in conjunction with rapid HIV testing and can be used to identify MSM at substantial risk for HIV infection who could benefit from additional prevention programs. The original PCC and its adapted versions can contribute to reducing HIV-related health disparities among high-risk MSM by raising awareness of and promoting reductions in personal risk behaviors.

\section{Acknowledgments}

Project ECHO study team: Erin Antunez, Phillip O. Coffin (Principal Investigator), Grant Colfax, Rand Dadasovich Moupali Das, Erin DeMicco, John Farley, Reggie Gage, Tim Matheson, Glenn-Milo Santos, Alic Shook of the Substance Use Research Unit, AIDS Office, San Francisco Department of Public Health, San Francisco, California; Kelly R. Knight, James W. Dilley, Eric Vittinghoff of the University of California, San Francisco, California. Vyann Howell and Phyllis Stoll at CDC contributed to the development of the PCC intervention package.

\section{References}

1. Purcell DW, Johnson CH, Lansky A, et al. Estimating the population size of men who have sex with men in the United States to obtain HIV and syphilis rates. Open AIDS J 2012;6(Suppl 1):98-107. http://dx.doi. org/10.2174/1874613601206010098.

2. CDC. Diagnoses of HIV in the United States and dependent areas, 2011. HIV Surveillance Report. Atlanta, GA: US Department of Health and Human Services, CDC; 2011. http://www.cdc.gov/hiv/pdf/ statistics_2011_HIV_Surveillance_Report_vol_23.pdf.

3. Johnson AS, Hall HI, Hu X, Lansky A, Holtgrave DR, Mermin J. Trends in diagnoses of HIV infection in the United States, 2002-2011. JAMA 2014;312:432-4. http://dx.doi.org/10.1001/jama.2014.8534.

4. CDC. Estimated HIV incidence in the United States, 2007-2010. In: HIV Surveillance Report Supplemental Report. Atlanta, GA: US Department of Health and Human Services, CDC; 2012. http://www. cdc.gov/hiv/pdf/statistics_hssr_vol_17_no_4.pdf.

5. CDC. Leading causes of death by age group, all males-United States, 2011. Atlanta, GA: US Department of Health and Human Services, CDC. http://www.cdc.gov/men/lcod/2011/lcod_menallages2011.pdf.

6. CDC. National HIV prevention progress report, 2013. Atlanta, GA: US Department of Health and Human Services, CDC; 2013. http:// www.cdc.gov/hiv/pdf/policies_NationalProgressReport.pdf.

7. Halkitis PN, Wolitski RJ, Millett GA. A holistic approach to addressing HIV infection disparities in gay, bisexual, and other men who have sex with men. Am Psychol 2013;68:261-73. http://dx.doi.org/10.1037/a0032746.

8. Bogart LM, Revenson TA, Whitfield KE, France CR. Introduction to the special section on lesbian, gay, bisexual, and transgender (LGBT) health disparities: where we are and where we're going. Ann Behav Med 2014;47:1-4. http://dx.doi.org/10.1007/s12160-013-9574-7.

9. Tao G, Hoover KW. Differences in access to healthcare and utilisation of HIV and sexually transmissible infection services between men who have sex with men and men who have sex only with women: results of the 2006-10 national survey of family growth in the United States. Sex Health 2013;10:363-8. http://dx.doi.org/10.1071/SH13017.

10. Paz-Bailey G, Pham H, Oster AM, et al. Engagement in HIV care among HIV-positive men who have sex with men from 21 cities in the United States. AIDS Behav 2014;18(Suppl 3):348-58. http://dx.doi. org/10.1007/s10461-013-0605-y.
11. Beer L, Oster AM, Mattson CL, Skarbinski J; Medical Monitoring Project. Disparities in HIV transmission risk among HIV-infected black and white men who have sex with men, United States, 2009. AIDS 2014;28:105-14. http://dx.doi.org/10.1097/QAD.0000000000000021.

12. Sullivan PS, Peterson J, Rosenberg ES, et al. Understanding racial HIV/ STI disparities in black and white men who have sex with men: a multilevel approach. PLoS One 2014;9:e90514. http://dx.doi. org/10.1371/journal.pone.0090514.

13. Gant Z, Bradley H, Hu X, Skarbinski J, Hall HI, Lansky A. Hispanics or Latinos living with diagnosed HIV: progress along the continuum of HIV care-United States, 2010. MMWR Morb Mortal Wkly Rep 2014;63:886-90.

14. Whiteside YO, Cohen SM, Bradley H, Skarbinski J, Hall HI, Lansky A. Progress along the continuum of HIV care among blacks with diagnosed HIV-United States, 2010. MMWR Morb Mortal Wkly Rep 2014;63:85-9.

15. Singh S, Bradley H, Hu X, Skarbinski J, Hall HI, Lansky A. Men living with diagnosed HIV who have sex with men: progress along the continuum of HIV care-United States, 2010. MMWR Morb Mortal Wkly Rep 2014;63:829-33.

16. McGarrity LA, Huebner DM. Is being out about sexual orientation uniformly healthy? The moderating role of socioeconomic status in a prospective study of gay and bisexual men. Ann Behav Med 2014;47:2838. http://dx.doi.org/10.1007/s12160-013-9575-6.

17. Herek GM. Hate crimes and stigma-related experiences among sexual minority adults in the United States: prevalence estimates from a national probability sample. J Interpers Violence 2009;24:54-74. http://dx.doi. org/10.1177/0886260508316477.

18. Huebner DM, Rebchook GM, Kegeles SM. Experiences of harassment, discrimination, and physical violence among young gay and bisexual men. Am J Public Health 2004;94:1200-3. http://dx.doi.org/10.2105/ AJPH.94.7.1200.

19. Hidalgo MA, Cotten C, Johnson AK, Kuhns LM, Garofalo R. 'Yes, I am more than just that': gay/bisexual young men residing in the United States discuss the influence of minority stress on their sexual risk behavior prior to HIV infection. Int J Sex Health 2013;25:291-304. http://dx.doi. org/10.1080/19317611.2013.818086.

20. Hatzenbuehler ML, McLaughlin KA. Structural stigma and hypothalamic-pituitary-adrenocortical axis reactivity in lesbian, gay, and bisexual young adults. Ann Behav Med 2014;47:39-47. http://dx.doi. org/10.1007/s12160-013-9556-9.

21. Mustanski B, Andrews R, Herrick A, Stall R, Schnarrs PW. A syndemic of psychosocial health disparities and associations with risk for attempting suicide among young sexual minority men. Am J Public Health 2014;104:287-94. http://dx.doi.org/10.2105/AJPH.2013.301744.

22. Irvin R, Wilton L, Scott $\mathrm{H}$, et al. A study of perceived racial discrimination in black men who have sex with men (MSM) and its association with healthcare utilization and HIV testing. AIDS Behav 2014;18:1272-8. http://dx.doi.org/10.1007/s10461-014-0734-y.

23. Gay and Lesbian Medical Association [Internet]. Washington, DC: Gay and Lesbian Medical Association; 2015. http://www.glma.org/index. cfm?fuseaction=Page.viewPage\&pageId= 532 .

24. Gonzales G, Blewett LA. National and state-specific health insurance disparities for adults in same-sex relationships. Am J Public Health 2014;104:e95-104. http://dx.doi.org/10.2105/AJPH.2013.301577.

25. McKirnan DJ, Du Bois SN, Alvy LM, Jones K. Health care access and health behaviors among men who have sex with men: the cost of health disparities. Health Educ Behav 2013;40:32-41. http://dx.doi.org/ $10.1177 / 1090198111436340$.

26. Woody GE, VanEtten-Lee ML, McKirnan D, et al.; HIVNET VPS 001 Protocol Team. Substance use among men who have sex with men: comparison with a national household survey. J Acquir Immune Defic Syndr 2001;27:8690. http://dx.doi.org/10.1097/00126334-200105010-00015. 
27. Ward BW, Dahlhamer JM, Galinsky AM, Joestl SS. Sexual orientation and health among U.S. adults: National Health Interview Survey, 2013. Natl Health Stat Report 2014;77:1-10.

28. Santos GM, Coffin PO, Das M, et al. Dose-response associations between number and frequency of substance use and high-risk sexual behaviors among HIV-negative substance-using men who have sex with men (SUMSM) in San Francisco. J Acquir Immune Defic Syndr 2013;63:540-4. http://dx.doi.org/10.1097/QAI.0b013e318293f10b.

29. Hirshfield S, Remien RH, Humberstone M, Walavalkar I, Chiasson MA. Substance use and high-risk sex among men who have sex with men: a national online study in the USA. AIDS Care 2004;16:1036-47. http://dx.doi.org/10.1080/09540120412331292525.

30. Vosburgh HW, Mansergh G, Sullivan PS, Purcell DW. A review of the literature on event-level substance use and sexual risk behavior among men who have sex with men. AIDS Behav 2012;16:1394-410. http:// dx.doi.org/10.1007/s10461-011-0131-8.

31. Buchbinder SP, VittinghoffE, Heagerty PJ, et al. Sexual risk, nitrite inhalant use, and lack of circumcision associated with HIV seroconversion in men who have sex with men in the United States. J Acquir Immune Defic Syndr 2005;39:82-9. http://dx.doi.org/10.1097/01.qai.0000134740.41585.f4.

32. Colfax GN, Mansergh G, Guzman R, et al. Drug use and sexual risk behavior among gay and bisexual men who attend circuit parties: a venue-based comparison. J Acquir Immune Defic Syndr 2001;28:373-9. http://dx.doi.org/10.1097/00126334-200112010-00011.

33. The White House Office of National AIDS Policy. National HIV/AIDS strategy for the United States. Washington, DC: The White House Office of National AIDS Policy; 2010. https://www.whitehouse.gov/sites/ default/files/uploads/NHAS.pdf.

34. CDC. Risk reduction. In: CDC. Compendium of evidence-based interventions and best practices for HIV prevention. Atlanta, GA: US Department of Health and Human Services, CDC; 2015. http://www. cdc.gov/hiv/prevention/research/compendium/rr/index.html.

35. Herbst JH, Beeker C, Mathew A, et al.; Task Force on Community Preventive Services. The effectiveness of individual-, group-, and community-level HIV behavioral risk-reduction interventions for adult men who have sex with men: a systematic review. Am J Prev Med 2007;32(Suppl):38-67. http://dx.doi.org/10.1016/j.amepre.2006.12.006.

36. Dilley JW, Woods WJ, Sabatino J, et al. Changing sexual behavior among gay male repeat testers for HIV: a randomized, controlled trial of a single-session intervention. J Acquir Immune Defic Syndr 2002;30:17786. http://dx.doi.org/10.1097/00042560-200206010-00006.

37. Dilley JW, Woods WJ, Loeb L, et al. Brief cognitive counseling with HIV testing to reduce sexual risk among men who have sex with men: results from a randomized controlled trial using paraprofessional counselors. J Acquir Immune Defic Syndr 2007;44:569-77. http:// dx.doi.org/10.1097/QAI.0b013e318033ffbd.

38. Dilley JW, Schwarcz S, Murphy J, Joseph C, VittinghoffE, Scheer S. Efficacy of personalized cognitive counseling in men of color who have sex with men: secondary data analysis from a controlled intervention trial. AIDS Behav 2011;15:970-5. http://dx.doi.org/10.1007/s10461-010-9771-3.

39. Schwarcz SK, Chen YH, Murphy JL, et al. A randomized control trial of personalized cognitive counseling to reduce sexual risk among HIVinfected men who have sex with men. AIDS Care 2013;25:1-10. http:// dx.doi.org/10.1080/09540121.2012.674095.

40. McPhee B, Skinta MD, Paul J, Dilley JW. Single-session personalized cognitive counseling to change HIV risk behavior among HIV-negative men who have sex with men: a two-part case study. Cognit Behav Pract 2012;19:328-37. http://dx.doi.org/10.1016/j.cbpra.2011.04.004.

41. Penman-Aguilar A, Boye K, Liburd L. Background and rationale. In: Strategies to reduce health disparities: selected CDC-sponsored interventions - United States, 2016. MMWR Suppl 2016;65(No. Suppl 1).
42. CDC. RFA-PS07-004: Adopting and demonstrating the adaptation of prevention techniques for persons at highest risk of acquiring or transmitting human immunodeficiency virus (ADAPT 2)-(UR6). Atlanta, GA: US Department of Health and Human Services, CDC; 2007. https:// grants.nih.gov/grants/guide/rfa-files/RFA-PS-07-004.html.

43. Knight KR, Das M, DeMicco E, et al. A roadmap for adapting an evidence-based HIV prevention intervention: personal cognitive counseling (PCC) for episodic substance-using men who have sex with men. Prev Sci 2014;15:364-75.

44. Coffin PO, Santos GM, Colfax G, et al. Adapted personalized cognitive counseling for episodic substance-using men who have sex with men: a randomized controlled trial. AIDS Behav 2014;18:1390-400. http:// dx.doi.org/10.1007/s10461-014-0712-4.

45. Santos GM, Coffin PO, Vittinghoff E, et al. Substance use and drinking outcomes in personalized cognitive counseling randomized trial for episodic substance-using men who have sex with men. Drug Alcohol Depend 2014;138:234-9. http://dx.doi.org/10.1016/j.drugalcdep.2014.02.015.

46. Branson BM, Handsfield HH, Lampe MA, et al. Revised recommendations for HIV testing of adults, adolescents, and pregnant women in healthcare settings. MMWR Recomm Rep 2006;55(No. RR-14).

47. Gossop M, Darke S, Griffiths P, et al. The severity of dependence scale (SDS): psychometric properties of the SDS in English and Australian samples of heroin, cocaine, and amphetamine users. Addiction 1995;90:607-14. http://dx.doi.org/10.1111/j.1360-0443.1995.tb02199.x.

48. CDC. Personalized cognitive risk-reduction counseling (with optional sex diary). In: CDC. Compendium of evidence-based interventions and best practices for HIV prevention. Atlanta, GA: US Department of Health and Human Services, CDC; 2013. http://www.cdc.gov/hiv/ prevention/research/compendium/rr/pcrrc.html.

49. Smith DK, Beltrami J. A proposed framework to monitor daily oral antiretroviral pre-exposure prophylaxis in the U.S. Am J Prev Med 2013;44(Suppl 2):S141-6. http://dx.doi.org/10.1016/j.amepre.2012.09.040.

50. Smith DK, Van Handel M, Wolitski RJ, et al. Vital signs: estimated percentages and numbers of adults with indications for preexposure prophylaxis to prevent HIV acquisition-United States, 2015. MMWR Morb Mortal Wkly Rep 2015;64:1291-5. http://dx.doi.org/10.15585/ mmwr.mm6446a4.

51. Neumann MS, Sogolow ED. Replicating effective programs: HIV/AIDS prevention technology transfer. AIDS Educ Prev 2000;12(Suppl):35-48.

52. University of California San Francisco, AIDS Health Project. Personalized cognitive counseling implementation manual. San Francisco, CA: University of California San Francisco; 2011. https://effectiveinterventions. cdc.gov/docs/default-source/pcc-docs/13-0204_PCC_Final_IM_ Appendices_and_Workbook.pdf?sfvrsn=0.

53. Danya International Inc. Effective interventions: HIV prevention that works. Atlanta, GA: Danya International Inc.; 2012. https:// effectiveinterventions.cdc.gov.

54. Danya International Inc. PCC: an overview for managers-online. Atlanta, GA: Danya International Inc.; 2014. https://effectiveinterventions.cdc. gov/en/TrainingCalendar/EventList/2014/07/10/default-calendar/ PCC_An_Overview_for_Managers_-_Online.

55. Mermin J, Fenton KA. The future of HIV prevention in the United States. JAMA 2012;308:347-8. http://dx.doi.org/10.1001/jama.2012.8693.

56. CDC. High-impact HIV prevention: CDC's approach to reducing HIV infections in the United States. Atlanta, GA: US Department of Health and Human Services, CDC; 2011. http://www.cdc.gov/hiv/pdf/ policies_NHPC_Booklet.pdf.

57. CDC, Division of HIV/AIDS Prevention. Turning the tide on HIV, annual report 2013. Atlanta, GA: US Department of Health and Human Services, CDC; 2014. http://www.cdc.gov/hiv/pdf/policies_DHAP_ AnnualReport_2013.pdf.

58. Collins C, Forsyth A, Gomez M, et al. Practical use of economic modeling and decision making to advance high-impact HIV prevention [Oral]. Presented at the US Conference on AIDS, New Orleans, LA; September 8-11, 2013. 
59. Eke AN, Mezoff JS, Duncan T, Sogolow ED. Reputationally strong HIV prevention programs: lessons from the front line. AIDS Educ Prev 2006;18:163-75. http://dx.doi.org/10.1521/aeap.2006.18.2.163.

60. Eaton LA, Huedo-Medina TB, Kalichman SC, et al. Meta-analysis of single-session behavioral interventions to prevent sexually transmitted infections: implications for bundling prevention packages. Am J Public Health 2012;102:e34-44. http://dx.doi.org/10.2105/AJPH.2012.300968.

61. Collins CB Jr, Edwards AE, Jones PL, Kay L, Cox PJ, Puddy RW. A comparison of the interactive systems framework (ISF) for dissemination and implementation and the CDC Division of HIV/AIDS Prevention's research-to-practice model for behavioral interventions. Am J Community Psychol 2012;50:518-29. http://dx.doi.org/10.1007/ s10464-012-9525-7.
62. Center of Excellence for Transgender Health; Center for AIDS Prevention Studies. Personalized cognitive counseling: an adaptation for working with trans women. San Francisco, CA: University of California San Francisco, Center of Excellence for Transgender Health and Center for AIDS Prevention Studies; 2012. https://effectiveinterventions.cdc.gov/ docs/default-source/pcc-docs/CoE_PCCAdaptation_3R.pdf?sfursn=0.

63. Sfhiv.org [Internet]. San Francisco, CA: San Francisco Department of Public Health; 2012. http://www.sfhiv.org.

64. San Francisco Department of Public Health. HIV epidemiology annual report, 2013. San Francisco, CA: San Francisco Department of Public Health, HIV Epidemiology Section; 2014. https://www.sfdph.org/dph/ files/reports/RptsHIVAIDS/HIVAIDAnnlRpt2013.pdf. 